\title{
Competing risk analyses of overall survival and cancer-specific survival in patients with combined hepatocellular cholangiocarcinoma after surgery
}

\author{
Chaobin $\mathrm{He}^{1 \dagger}$, Yu Zhang ${ }^{2 \dagger}$, Zhiyuan $\mathrm{Cai}^{1}$ and Xiaojun Lin $^{1 *} \mathrm{C}$
}

\begin{abstract}
Background: Our objective was to identify risk factors affecting overall survival (OS) and cancer-specific survival (CSS) and build nomograms to predict survival based on a large population-based cohort.

Methods: Two hundred and thirty patients diagnosed with CHCC between 2004 and 2015 were retrospectively extracted from the Surveillance, Epidemiology, and End Results (SEER) database as a training cohort. In addition, Ninety-nine patients diagnosed with CHCC between 2000 and 2017 were retrospectively extracted from Sun Yat-Sen University Cancer Center (SYSUCC) as an external validation. Nomograms for predicting probability of OS and CSS were established. Performance of the nomograms was measured by concordance index (C-index) and the area under receiver operating characteristic (ROC) curve (AUC).

Results: In training cohort, the 1-, 2 and 3-year OS were 67.7, 46.8 and 37.9\%, and the 1-, 2 and 3-year CSS were $73.1,52.0$ and $43.0 \%$, respectively. The established nomograms were well calibrated in both training and validation cohort, with concordance indexes (C-index) of 0.652 and 0.659 , respectively for OS prediction; 0.706 and 0.763 , respectively for CSS prediction. Nomograms also displayed better discriminatory compared with 8th edition tumor-node-metastasis (TNM) stage system for predicting OS and CSS.

Conclusion: We constructed nomograms to predict OS and CSS based on a relatively large cohort. The established nomograms were well validated and could serve to improve predictions of survival risks and guide management of patients with CHCC after surgery.
\end{abstract}

Keywords: Combined hepatocellular cholangiocarcinoma, Overall survival, Cancer-specific survival, Nomogram, Prognosis

\section{Background}

Combined hepatocellular cholangiocarcinoma (CHCC) is a rare primary liver cancer, which is composed of mixed elements of both hepatocellular carcinoma (HCC) and cholangiocarcinoma (ICC) [1] and accounts for only $0.4-14.5 \%$ of the primary liver cancer [2]. Regarding the treatment of $\mathrm{CHCC}$, patients can obtain the best chance

\footnotetext{
* Correspondence: linxj@sysucc.org.cn

${ }^{\dagger}$ Chaobin He and Yu Zhang contributed equally to this work.

'Department of Hepatobiliary and Pancreatic Surgery, State Key Laboratory of Oncology in South China, Collaborative Innovation Center for Cancer Medicine, Sun Yat-sen University Cancer Center, Guangzhou 510060, China Full list of author information is available at the end of the article
}

to the greatest survival benefit from surgery [3]. However, CHCC has worse prognosis compared with HCC or ICC [4]. CHCC was firstly described in 1949 by Allen and Lisa [5]. However, due to the low morbidity of $\mathrm{CHCC}$ and the absence of unified diagnostic criterion, the clinical and pathological features of CHCC remain unclear. Moreover, different from HCC for which many preoperative prognostic prediction systems have been established [6-8], the prognostic stage system of patients with $\mathrm{CHCC}$ remained unclear, varying considerably from different reports [9-11]. The 8th tumor-node-metastasis (TNM) stage system, although it was the most frequently used stage system, it contained some common 
prognostic factors, such as tumor size, lymph node (LN) metastasis and distant metastasis. In addition, there was no a TNM stage system which is specially designed for CHCC. It was reported that the differences of clinical features among $\mathrm{CHCC}, \mathrm{HCC}$ and ICC could lead to the variations of prognostic factors $[12,13]$. There were also many factors, such as age, gender and tumor grade, which were shown to have great impact on survival. However, they were not included in the TNM stage system. Therefore, it is necessary to establish a stage system which is specially designed for prognostic prediction in patients with $\mathrm{CHCC}$.

In addition, with the improvement of survival of cancer patients, most of patients with $\mathrm{CHCC}$ are faced with advanced ages, which are associated with an increasing high rate of comorbidities. Moreover, ta high risk of competing events, which might contribute to more competing deaths, was observed in patients with $\mathrm{CHCC}$ as the age increases [14, 15]. Thus, when prognosis is evaluated, competing risks are worthy of being considered. However, most prognostic analyses only focused on overall survival (OS) and ignored the impact of survival from competing events $[8,9,16]$. Competing risk analysis evaluates the informative nature of censoring and the occurrence rates of a particular event, which is more suitable for prognostic analysis. Misleading conclusions might be obtained due to the failure to recognize the presence of competing risks in survival analysis [17].

The present study was to build nomograms to predict 1-, 2-, and 3-year OS and cancer-specific survival (CSS) of these patients based on the Surveillance, Epidemiology, and End Results (SEER) database. Also, another large cohort of patients with CHCC from China was used to externally validate the established nomograms.

\section{Methods \\ Patients}

The study population was identified from SEER database from 2004 to 2015 . We focused on cases pathologically confirmed CHCC after surgery [International Classification of Diseases for Oncology, Third Edition (ICD-O-3) site code C22.0 and C22.1; histology code: $8180 / 3$ ]. In addition, consecutive patients with pathological diagnosis of $\mathrm{CHCC}$ after surgery between 2000 and 2017 at the department of Hepatobiliary and Pancreatic Surgery of Sun Yat-Sen University Cancer Center (SYSUCC) were also enrolled in the present study. The exclusion criteria are the same as those described in our previous study [10].

Table 1 The comparison of clinicopathological factors between training cohort and validation cohort

\begin{tabular}{|c|c|c|c|c|c|}
\hline \multirow[t]{2}{*}{ Characteristic } & & \multirow[t]{2}{*}{$\mathrm{N}$} & \multicolumn{2}{|l|}{ Patients } & \multirow[t]{2}{*}{$P$} \\
\hline & & & Training cohort & $\overline{\text { Validation cohort }}$ & \\
\hline Total & & 329 & 230 & 99 & \\
\hline Age (median, years) & & & $60.5 \pm 10.41$ & $50.0 \pm 11.15$ & \\
\hline \multirow[t]{2}{*}{ Gender } & Male & 234 & 161 & 73 & 0.511 \\
\hline & Female & 95 & 69 & 26 & \\
\hline \multirow[t]{2}{*}{ Tumor site } & Intrahepatic biliary tract & 29 & 16 & 13 & 0.089 \\
\hline & Liver & 300 & 214 & 86 & \\
\hline \multirow[t]{3}{*}{ Tumor grade } & Well & 8 & 8 & 0 & 0.058 \\
\hline & Moderate & 143 & 92 & 51 & \\
\hline & Poor & 178 & 130 & 48 & \\
\hline \multirow[t]{2}{*}{ Tumor size } & $\leq 5 \mathrm{~cm}$ & 183 & 137 & 46 & 0.056 \\
\hline & $>5 \mathrm{~cm}$ & 146 & 93 & 53 & \\
\hline \multirow[t]{4}{*}{ T stage (8th) } & I & 137 & 93 & 44 & 0.203 \\
\hline & $\|$ & 108 & 83 & 25 & \\
\hline & III & 62 & 38 & 24 & \\
\hline & IV & 22 & 16 & 6 & \\
\hline \multirow[t]{2}{*}{ LN metastasis } & Absent & 291 & 204 & 87 & 0.852 \\
\hline & Present & 38 & 26 & 12 & \\
\hline \multirow[t]{3}{*}{ TNM stage (8th) } & I & 122 & 79 & 43 & 0.509 \\
\hline & $\|$ & 104 & 77 & 27 & \\
\hline & III & 103 & 74 & 29 & \\
\hline
\end{tabular}




\section{Data collection}

Records for the age at diagnosis, gender, tumor site, tumor grade, tumor size, TNM stage, follow-up information and cause of death were retrospectively retrieved from SEER database and the medical management system of SYSUCC. Survival time was defined as the duration from the date of diagnosis to last follow-up or death due to all causes (OS) or CHCC (CSS).

\section{Nomogram construction and validation}

Nomograms were constructed based on cohort from SEER database and externally validated based on cohort from SYSUCC database. Student's $t$ test and chi-square test or Fisher's exact test were used to compare continuous variables and categorical variables, respectively. The Kaplan-Meier curves were analyzed by log-rank tests. Univariate analysis and multivariate analysis were constructed using the Cox regression model and hazard ratio (HR) and the associated 95\% confidence interval (CI) for each variable were determined. Clinical and pathological factors were analyzed by the Fine and Grey's model for their cumulative incidence function (CIF) on cancer-specific mortality and non-cancer-specific mortality. Independent prognostic factors identified in the multivariate analysis were used to build nomograms to predict the 1-, 2- and 3-year OS and CSS rates.

As two important aspects of the performance of the established nomograms, the discrimination and calibration power were evaluated by concordance index (C-index) and calibration curves, respectively [18]. Bootstraps with 1000 resamples were used in the validation of the nomogram. In addition, the area under receiver operating characteristic (ROC) curve (AUC) was used to evaluate the precision of the survival predictions.

$\mathrm{R}$ version 3.4.2 software (The R Foundation for Statistical Computing, Vienna, Austria. http://www.r-project.org), along with SPSS version 22 (SPSS Inc., Chicago, IL, USA), was used to conduct statistical analyses. A two tailed $P$-value $<0.05$ was considered statistically significant.

\section{Results}

Patient characteristics

Two hundred and thirty patients with $\mathrm{CHCC}$ and another ninety-nine patients with $\mathrm{CHCC}$ were retrospectively

Table 2 Overall survival rates and cumulative incidences of mortality among patients with CHCC after surgery

\begin{tabular}{|c|c|c|c|c|c|c|c|c|c|c|c|c|c|c|c|}
\hline \multirow[t]{2}{*}{ Characteristic } & & \multicolumn{2}{|c|}{ Patients } & \multicolumn{3}{|c|}{ Overall survival rate (\%) } & \multirow[t]{2}{*}{$P$} & \multicolumn{3}{|c|}{$\begin{array}{l}\text { Cancer-specific } \\
\text { mortality (\%) }\end{array}$} & \multirow[t]{2}{*}{$P$} & \multicolumn{3}{|c|}{$\begin{array}{l}\text { Non-cancer-specific } \\
\text { mortality (\%) }\end{array}$} & \multirow[t]{2}{*}{$P$} \\
\hline & & No. & $\%$ & 1-year & 2-year & 3-year & & 1-year & 2-year & 3-year & & 1-year & 2-year & 3-year & \\
\hline Total & & 230 & 100 & 67.7 & 46.8 & 37.9 & & 25.9 & 45.2 & 53.4 & & 5.9 & 8.0 & 8.0 & \\
\hline \multirow[t]{2}{*}{ Age (years) } & $\leq 60$ & 115 & 50 & 71.8 & 50.2 & 46.9 & 0.014 & 21.1 & 40.8 & 44.2 & 0.007 & 6.4 & 8.5 & 8.5 & 0.442 \\
\hline & $>60$ & 115 & 50 & 63.5 & 42.8 & 28.4 & & 30.8 & 49.9 & 63.2 & & 5.5 & 7.6 & 7.6 & \\
\hline \multirow[t]{2}{*}{ Gender } & Male & 161 & 70 & 64.5 & 44.4 & 39.3 & 0.742 & 27.2 & 45.2 & 50.4 & 0.389 & 7.9 & 10.1 & 10.1 & 0.051 \\
\hline & Female & 69 & 30 & 74.9 & 51.5 & 35.3 & & 22.9 & 45.3 & 60.0 & & 1.5 & 3.3 & 3.3 & \\
\hline \multirow[t]{2}{*}{ Tumor site } & Intrahepatic biliary tract & 16 & 7 & 93.3 & 77.5 & 77.5 & 0.019 & 6.7 & 22.5 & 22.5 & 0.073 & 0 & 0 & 0 & 0.222 \\
\hline & Liver & 214 & 93 & 65.8 & 44.4 & 35.5 & & 27.4 & 46.8 & 55.2 & & 6.4 & 8.6 & 8.6 & \\
\hline \multirow[t]{3}{*}{ Tumor grade } & Well & 8 & 3 & 80.0 & 80.0 & 60.0 & 0.016 & 20.0 & 20.0 & 40.0 & 0.055 & 0 & 0 & 0 & 0.720 \\
\hline & Moderate & 92 & 40 & 76.4 & 55.1 & 50.7 & & 17.1 & 36.2 & 40.6 & & 6.5 & 8.7 & 8.7 & \\
\hline & Poor & 130 & 57 & 59.4 & 35.5 & 23.7 & & 31.0 & 53.2 & 63.6 & & 8.5 & 11.5 & 11.5 & \\
\hline \multirow[t]{2}{*}{ Tumor size } & $\leq 5 \mathrm{~cm}$ & 137 & 60 & 74.8 & 55.4 & 51.2 & 0.041 & 16.0 & 33.1 & 37.4 & 0.003 & 8.4 & 10.9 & 10.9 & 0.104 \\
\hline & $>5 \mathrm{~cm}$ & 93 & 40 & 65.9 & 43.6 & 30.3 & & 30.4 & 51.8 & 63.3 & & 3.1 & 4.9 & 4.9 & \\
\hline \multirow[t]{4}{*}{ T stage (8th) } & I & 93 & 40 & 82.4 & 62.3 & 55.7 & 0.015 & 11.3 & 29.9 & 34.3 & 0.005 & 6.6 & 8.5 & 8.5 & 0.568 \\
\hline & $\|$ & 83 & 36 & 69.5 & 46.2 & 39.6 & & 44.5 & 42.4 & 46.7 & & 6.8 & 10.6 & 10.6 & \\
\hline & III & 38 & 17 & 55.2 & 45.8 & 23.8 & & 37.7 & 47.0 & 69.1 & & 7.1 & 7.1 & 7.1 & \\
\hline & IV & 16 & 7 & 57.1 & 22.9 & 11.4 & & 37.7 & 75.1 & 75.1 & & 0 & 0 & 0 & \\
\hline \multirow[t]{2}{*}{ LN metastasis } & Absent & 204 & 89 & 70.1 & 48.4 & 39.6 & 0.253 & 24.2 & 44.9 & 53.1 & 0.887 & 5.5 & 6.7 & 6.7 & 0.142 \\
\hline & Present & 26 & 11 & 52.0 & 40.4 & 27.0 & & 36.0 & 41.8 & 48.5 & & 12.0 & 17.8 & 17.8 & \\
\hline \multirow[t]{3}{*}{ TNM stage (8th) } & 1 & 79 & 34 & 81.5 & 62.7 & 55.4 & 0.014 & 11.3 & 32.5 & 37.4 & 0.084 & 5.6 & 5.6 & 5.6 & 0.896 \\
\hline & $\|$ & 63 & 27 & 74.9 & 53.7 & 51.0 & & 18.0 & 39.0 & 41.6 & & 2.2 & 7.3 & 7.3 & \\
\hline & III & 88 & 39 & 55.2 & 40.0 & 27.3 & & 35.7 & 48.0 & 60.6 & & 9.2 & 12.1 & 12.1 & \\
\hline
\end{tabular}


identified from SEER database as training cohort and SYSUCC database as external validation cohort, respectively in the present study. The baseline characteristics of the training cohort and the validation cohort were shown in Table 1. Among these patients, the mean age was 59.8 years and 49.7 years for the patients in the training cohort and validation cohort, respectively. Most patients were male and had tumor origin from liver in both train and validation cohort. Poor differentiation $(130,56.7 \%)$ was the most common tumor grade, while most patients had tumors which were moderately differentiated in the validation cohort. The proportions of patients were comparable between two cohorts in terms of $\mathrm{T}$ stage (8th), $\mathrm{N}$ stage (8th) and 8th edition TNM stage system.

\section{OS and CSS of patients}

During the follow-up period, deaths were observed in 142 out of $230(61.7 \%)$ patients in the training cohort and 43 out of $99(43.4 \%)$ patients in the validation cohort. In the training cohort, $\mathrm{CHCC}$ contributed to deaths of 118 (51.3\%) patients and competing risk events contributed to deaths of $24(10.4 \%)$ patients. In the validation cohort, there were 31 cancer-specific death and 12 non-cancer-specific death during the follow-up period. Table 2 outlined the comparisons of 1-, 2- and 3 -year OS rates, cancer-specific mortalities and non-cancer-specific mortalities of patients. It was shown that older age, larger tumor and advanced $\mathrm{T}$ stage (8th) were responsible for higher cumulative rates of cancer-specific-mortality. Earlier $\mathrm{N}$ stage (8th), well differentiation and
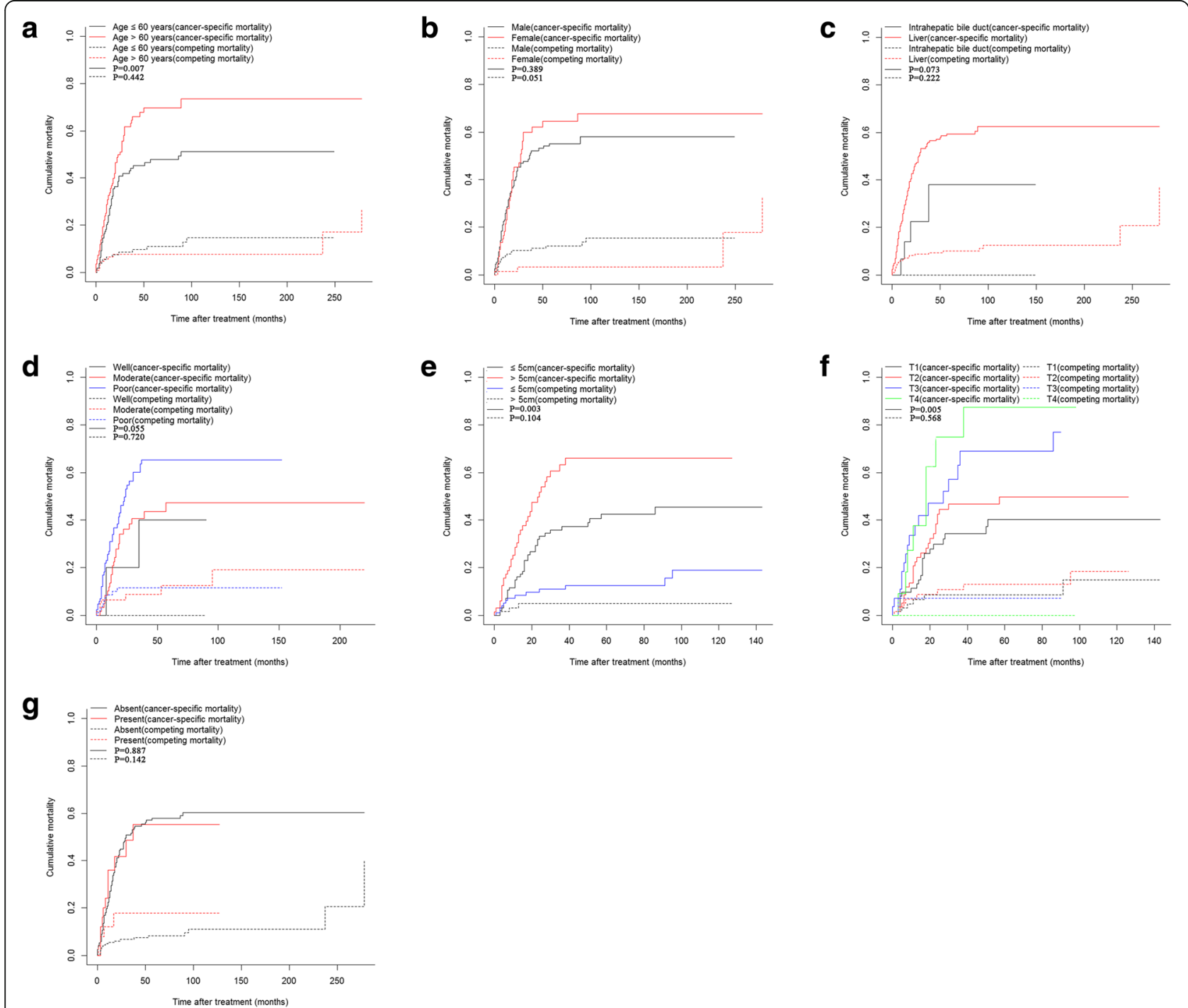

Fig. 1 Cumulative cancer-specific and competing mortality according to patient characteristics: (a) Age; (b) Gender; (c) Tumor site; (d) Tumor grade; (e) Tumor size; (f) T stage (8th); (g) N stage (8th). Abbreviations: LN, lymph node 
origin from intrahepatic bile duct seemed to be related to the decreased cancer-special-mortalities while the differences were not significant (Fig. 1).

The median OS and CSS for patients were 22.0 (95\% CI: 18.0-29.0) months and 27.0 (95\%CI: 20.0-37.0) months, respectively. The 1-, 2 and 3-year OS were 67.7, 46.8 and $37.9 \%$, and the 1-, 2 and 3-year CSS were 73.1, 52.0 and $43.0 \%$, respectively. The Kaplan-Meier curves of OS analyses were shown in Fig. 2. Patients who were younger than 60 years old or had smaller tumor $(\leq 5 \mathrm{~cm})$ had significant longer OS. Tumor originated from intrahepatic bile ducts, well differentiated tumor, or earlier $\mathrm{T}$ stage (8th) also indicated better OS.

\section{Construction and validation of nomograms}

Univariate analyses were performed to filter prognostic factors. It was revealed that age, tumor site, tumor grade, tumor size and $\mathrm{T}$ stage (8th) were significantly associated with OS. After a stepwise removal of variables, age (HR $=1.031,95 \% \mathrm{CI}=1.006-1.056, P=0.015)$, tumor grade
$(\mathrm{HR}=2.049,95 \% \mathrm{CI}=1.277-3.288, \quad P=0.003)$ and $\mathrm{T}$ stage (8th) $(\mathrm{HR}=1.410,95 \% \mathrm{CI}=1.071-1.855, P=0.014)$ remained significant predictors for OS (Table 3). Proportional subdistribution hazard assumption for CSS analysis also showed that Age $(\mathrm{HR}=1.038,95 \% \mathrm{CI}=$ $1.010-1.067, P=0.008)$, tumor grade $(\mathrm{HR}=2.027,95 \%$ $\mathrm{CI}=1.195-3.439, \quad P=0.009)$, tumor size $(\mathrm{HR}=1.849$, 95\% $\mathrm{CI}=1.001-3.427, P=0.049)$ and $\mathrm{T}$ stage (8th) (HR $=1.429,95 \% \mathrm{CI}=1.038-1.969, P=0.029)$ were all independently associated with CSS.

Nomograms for predicting OS and CSS were constructed with all of the independent predictors of patients in the training cohort (Fig. 3). The C-indexes for OS and CSS prediction were $0.652(95 \% \mathrm{CI}=0.579$ $0.725)$ and $0.706(95 \% \mathrm{CI}=0.630-0.782)$, respectively, showing good accuracy of the established nomograms for survival prediction. In addition, the comparison of C-indexes of the established nomograms and the 8th edition TNM stage system showed that the established nomograms had enhanced discriminatory ability in a

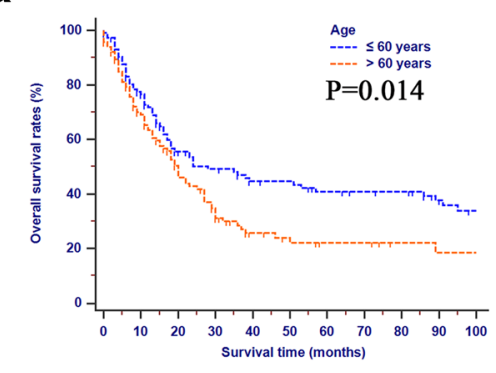

d

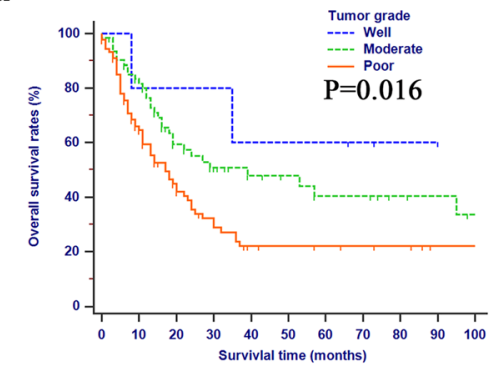

$\mathbf{g}$

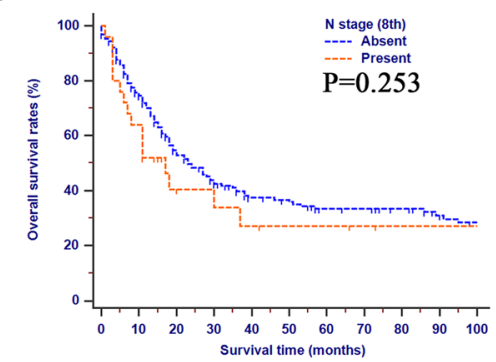

b

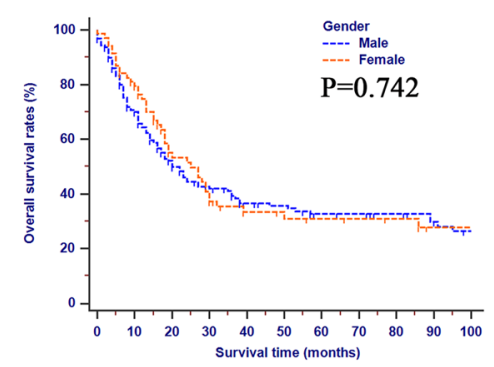

e

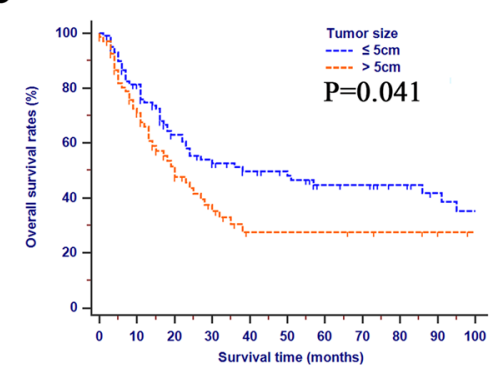

C

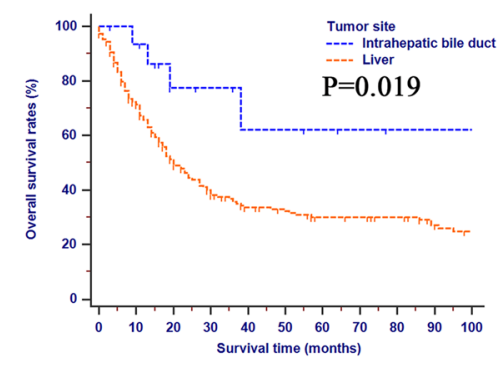

f

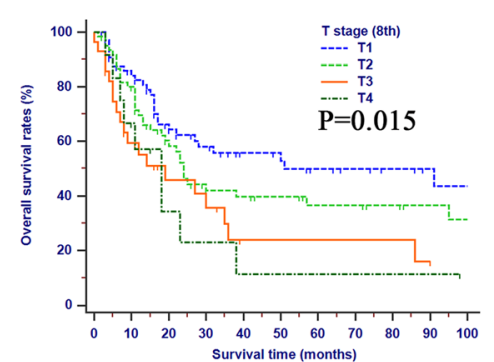

Fig. 2 Overall survival rates according to clinical and pathological characteristics: (a) Age; (b) Gender; (c) Tumor site; (d) Tumor grade; (e) Tumor size; (f) T stage (8th); (g) N stage (8th). Abbreviations: LN, lymph node 


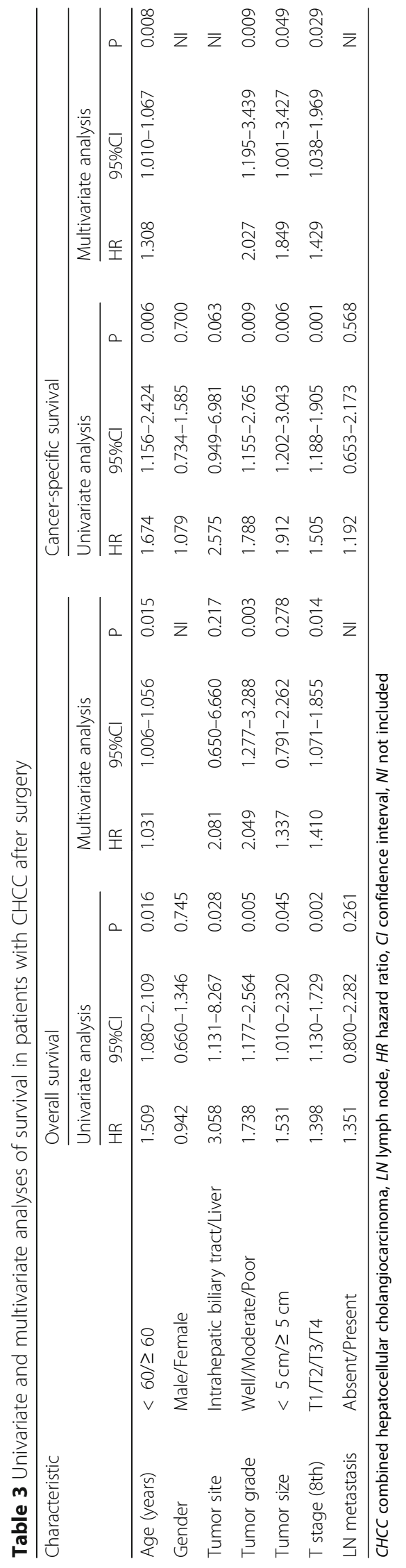




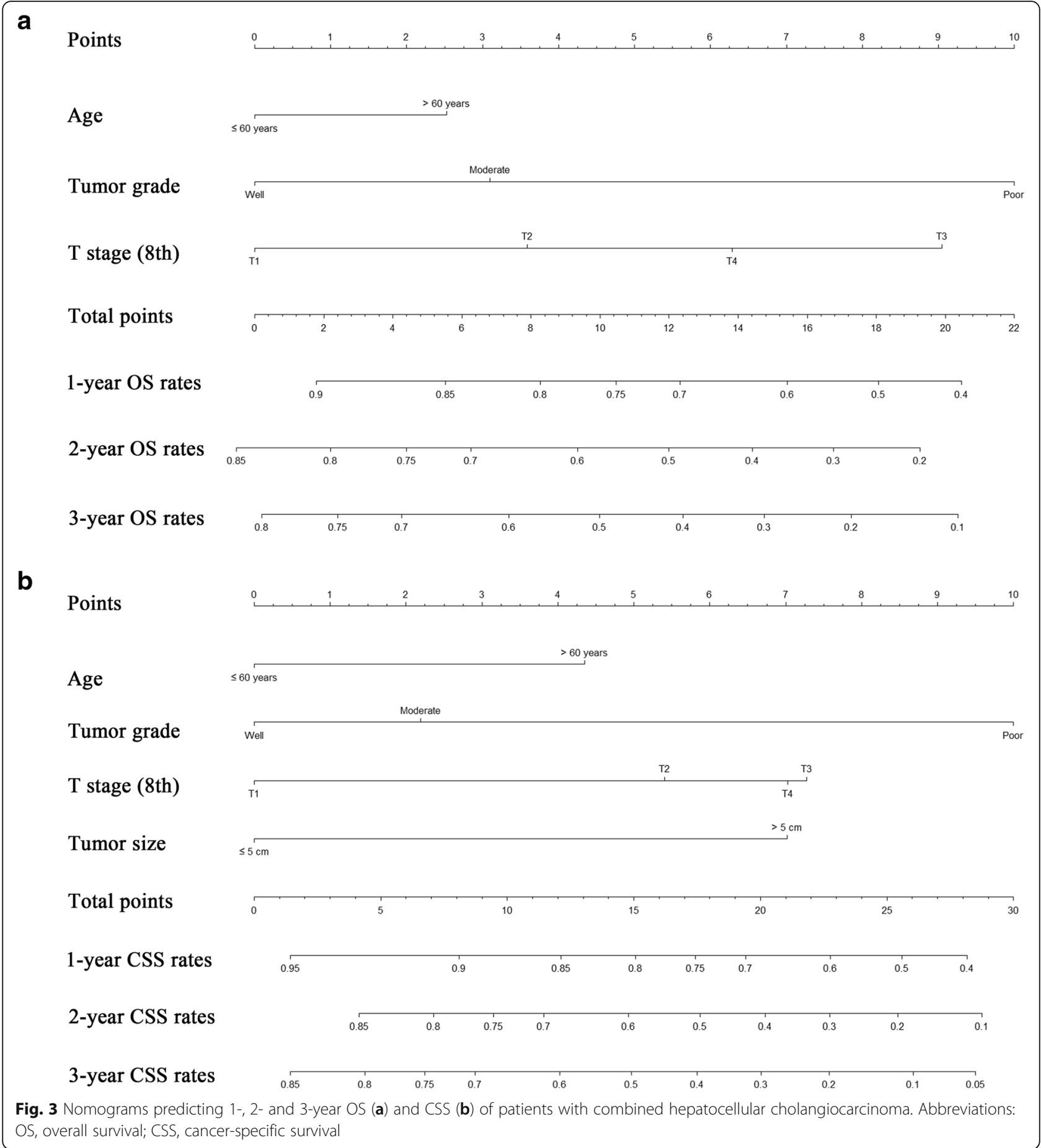

predicting OS and CSS (OS, C-index $=0.652,95 \% \mathrm{CI}=$ $0.579-0.725$ vs $\mathrm{C}$-index $=0.567,95 \% \mathrm{CI}=0.492-0.642, \mathrm{P}$ $=0.015 ;$ CSS, C-index $=0.706,95 \% \mathrm{CI}=0.630-0.782$ vs C-index $=0.553,95 \% \mathrm{CI}=0.469-0.637, \quad P<0.001)$. The accuracy of nomogram was verified by bootstrapped resamples via the validation cohort. Fair agreement between the nomogram-predicted survival and the actual survival was observed (Fig. 4) and it was indicated that discrimination of nomogram with regard to the SYSUCC validation cohort was also higher than that of 8th edition TNM stage system even though it did not exhibit independent significance (Table 4).

Furthermore, two ROC models of OS and CSS regarding the prediction ability were compared (Table 5). In the training cohort, the values of AUC of the nomogram for predicting 1-, 2 and 3-year OS and CSS were 0.703, 

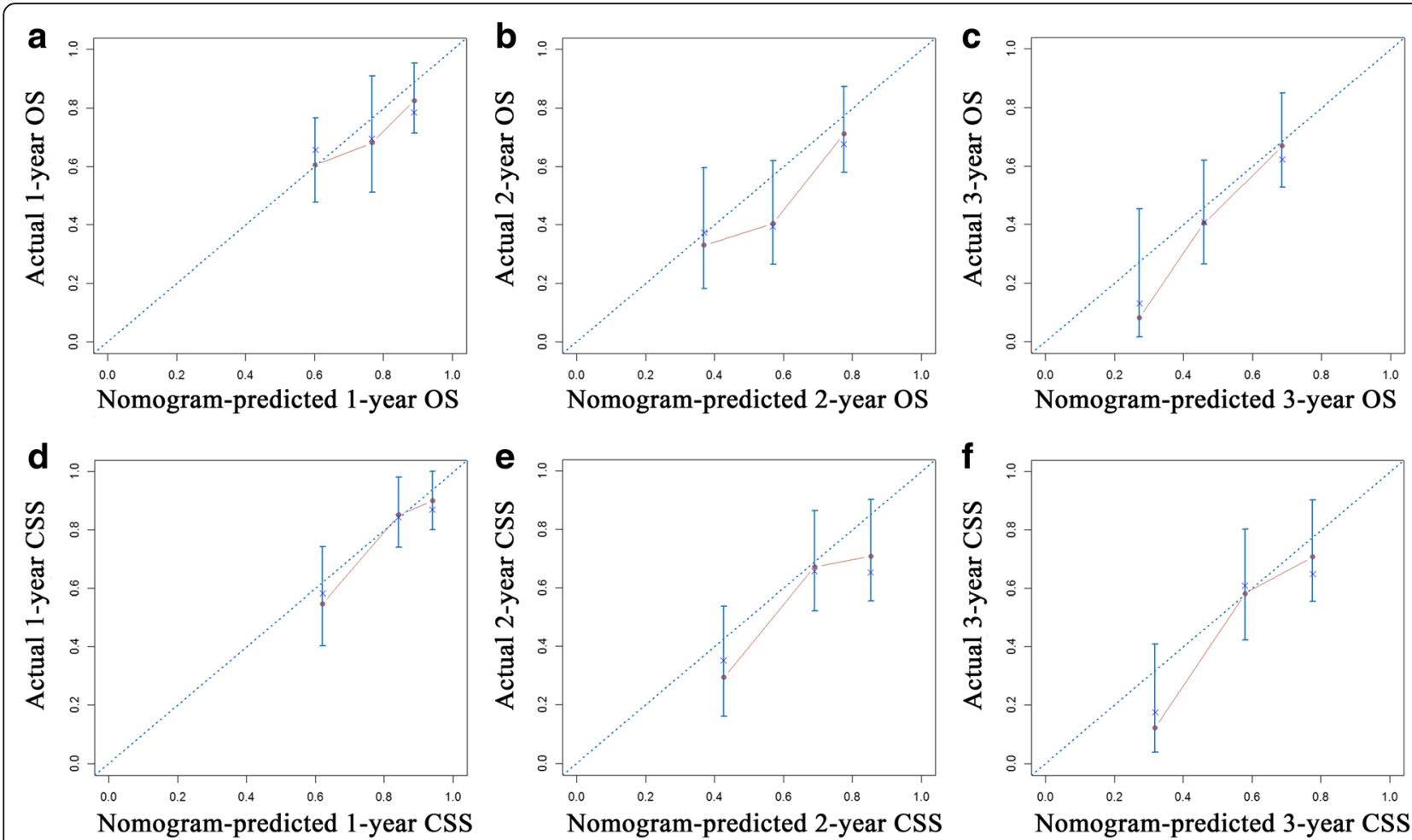

Fig. 4 Calibration plots of the nomogram for 1-, 2- and 3-year OS (a, b, c) and CSS (d, e, f) prediction. X-axis represents the nomogram-predicted probability of survival; Y-axis represents the actual OS probability. A perfectly accurate nomogram prediction model would result in a plot that the observed and predicted probabilities for given groups fall along the 45-degree line. Dots with bars represent nomogram-predicted probabilities along with 95\% confidence interval. Abbreviations: OS, overall survival; CSS, cancer-specific survival

0.675 and $0.753 ; 0.752,0.702$ and 0.791 , respectively, which were all higher than those of 8th edition TNM stage system (Fig. 5). Regarding to the validation cohort, the values of AUC of the nomogram for predicting 1-, 2 and 3-year OS and CSS were 0.638, 0.647 and 0.600; $0.775,0.800$ and 0.785 , respectively, whereas the AUC values of the 8th edition TNM stage system for predicting 1-, 2 and 3-year OS and CSS were 0.630, 0.638 and $0.575 ; 0.722,0.720$ and 0.689 , respectively (Fig. 6). The established nomograms showed superior discriminatory capacity than 8th TNM stage system for predicting OS and CSS in both training and validation cohort.

\section{Discussion}

$\mathrm{CHCC}$ is a primary malignant tumor and represents a small proportion of all liver cancers. Due to the rarity of
$\mathrm{CHCC}$, most previous studies of $\mathrm{CHCC}$ were only limited to single-center cohorts with small sample sizes. The clinicopathological predictors of $\mathrm{CHCC}$ remained unclear and the special predictive system was unavailable for the personal treatment. Moreover, most previous studies mainly focused on OS, other than CSS, which reflected the nature of causes of deaths in cancer patients, especially those with increasing ages [19]. Thus, we tried to evaluate the mortality of patients and built nomograms to predict OS and CSS for patients with $\mathrm{CHCC}$ after surgery in this study.

It was observed that the increasing ages had a negative effect of survival in patients with CHCC after surgery, which was more obvious on CSS than OS. Moreover, similar with other studies [20,21], it was indicated that the increasing ages were shown to be independent

Table 4 C-indexes for the nomograms and TNM staging systems in patients with PC after IRE treatment

\begin{tabular}{|c|c|c|c|c|c|}
\hline Survival & & Training set & $P$ & Validation set & $P$ \\
\hline \multirow[t]{2}{*}{ Overall survival } & Nomogram & $0.652(0.579-0.725)$ & Reference & $0.659(0.571-0.747)$ & Reference \\
\hline & TNM 8th stage & $0.567(0.492-0.642)$ & 0.015 & $0.592(0.505-0.679)$ & 0.088 \\
\hline \multirow[t]{2}{*}{ Cancer-specific survival } & Nomogram & $0.706(0.630-0.782)$ & Reference & $0.763(0.689-0.837)$ & Reference \\
\hline & TNM 8th stage & $0.553(0.469-0.637)$ & $<0.001$ & $0.684(0.603-0.765)$ & 0.073 \\
\hline
\end{tabular}


Table 5 Values of AUR for the nomograms and TNM staging systems in patients with PC after IRE treatment

\begin{tabular}{|c|c|c|c|c|c|c|c|}
\hline \multirow[t]{2}{*}{ Survival } & & \multicolumn{3}{|c|}{ Training set } & \multicolumn{3}{|c|}{ Validation set } \\
\hline & & 1-year & 2-year & 3-year & 1-year & 2-year & 3-year \\
\hline \multirow[t]{2}{*}{ Overall survival } & Nomogram & 0.703 & 0.675 & 0.753 & 0.638 & 0.647 & 0.600 \\
\hline & TNM 8th stage & 0.623 & 0.549 & 0.633 & 0.630 & 0.638 & 0.575 \\
\hline \multirow[t]{2}{*}{ Cancer-specific survival } & Nomogram & 0.752 & 0.702 & 0.791 & 0.775 & 0.800 & 0.785 \\
\hline & TNM 8th stage & 0.628 & 0.530 & 0.628 & 0.722 & 0.720 & 0.689 \\
\hline
\end{tabular}

AUC area under ROC curve; other abbreviations as in Table S1

prognostic factors of survival in this study. Thus, maybe considering age was more appropriate when prognosis of patients with $\mathrm{CHCC}$ after surgery was evaluated.

In the presence of competing risk model, other independent prognostic factors included tumor grade, tumor size and $\mathrm{T}$ stage (8th). Tumor size is the predominant feature of $\mathrm{T}$ stage (8th) and an important component of the 8th edition TNM stage system. It was shown that advanced $T$ stage (8th) represented greater risks of lower OS and CSS in this study. In addition, heavier weight from $T$ stage (8th) in predicting CSS than OS was observed, showing cancer-specific mortalities were more largely depended on inherent feature of tumor. Another factor reflected the intrinsic nature of tumor, tumor grade, was also associated with changes of prognoses of patients with $\mathrm{CHCC}$, which was in accordance with many previous studies [12, 22, 23]. The addition of tumor grade, which was independent of other prognostic factors, such as tumor size and LN metastasis, might contribute to more accurate estimation of tumor behavior and survival outcomes of patients [24].

The differences of origin and the complex nature may lead to the unique features of $\mathrm{CHCC}$ compared with $\mathrm{HCC}$ and ICC. The predictive significance was not observed for $\mathrm{LN}$ metastasis in patients with $\mathrm{CHCC}$ in this study. This result was similar with that from a large-scale study [25]. The proportion of patients who were accompanied with LN metastasis was extremely low. In this study, LN metastasis was depended on surgical resection and pathologic confirmation, other than imaging scan. This criterion could contribute to the lower rates of LN metastasis. In addition, similar with other similar studies [23, 25], as an important indicator of advanced TNM stages, LN metastasis was failed to

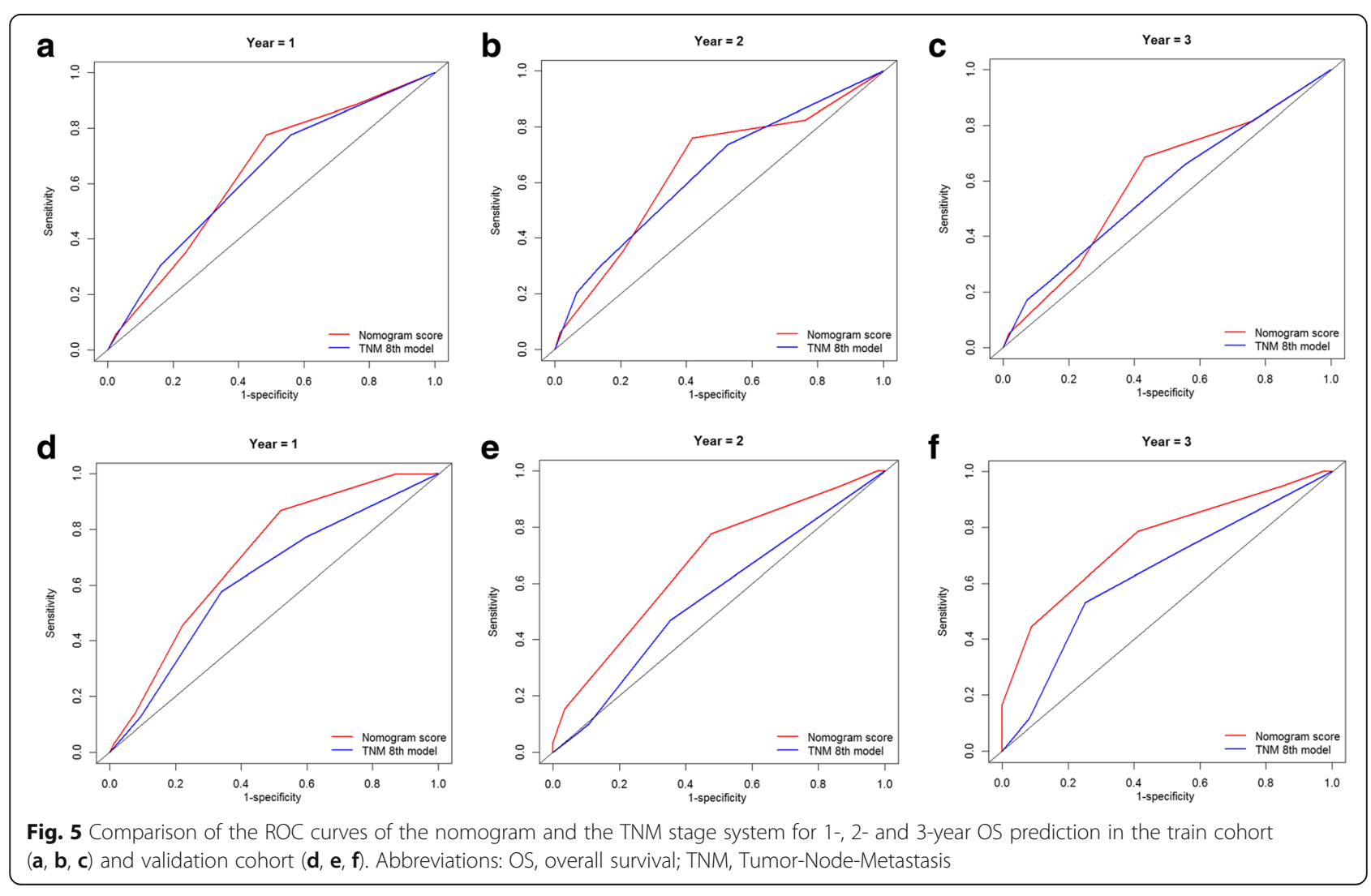



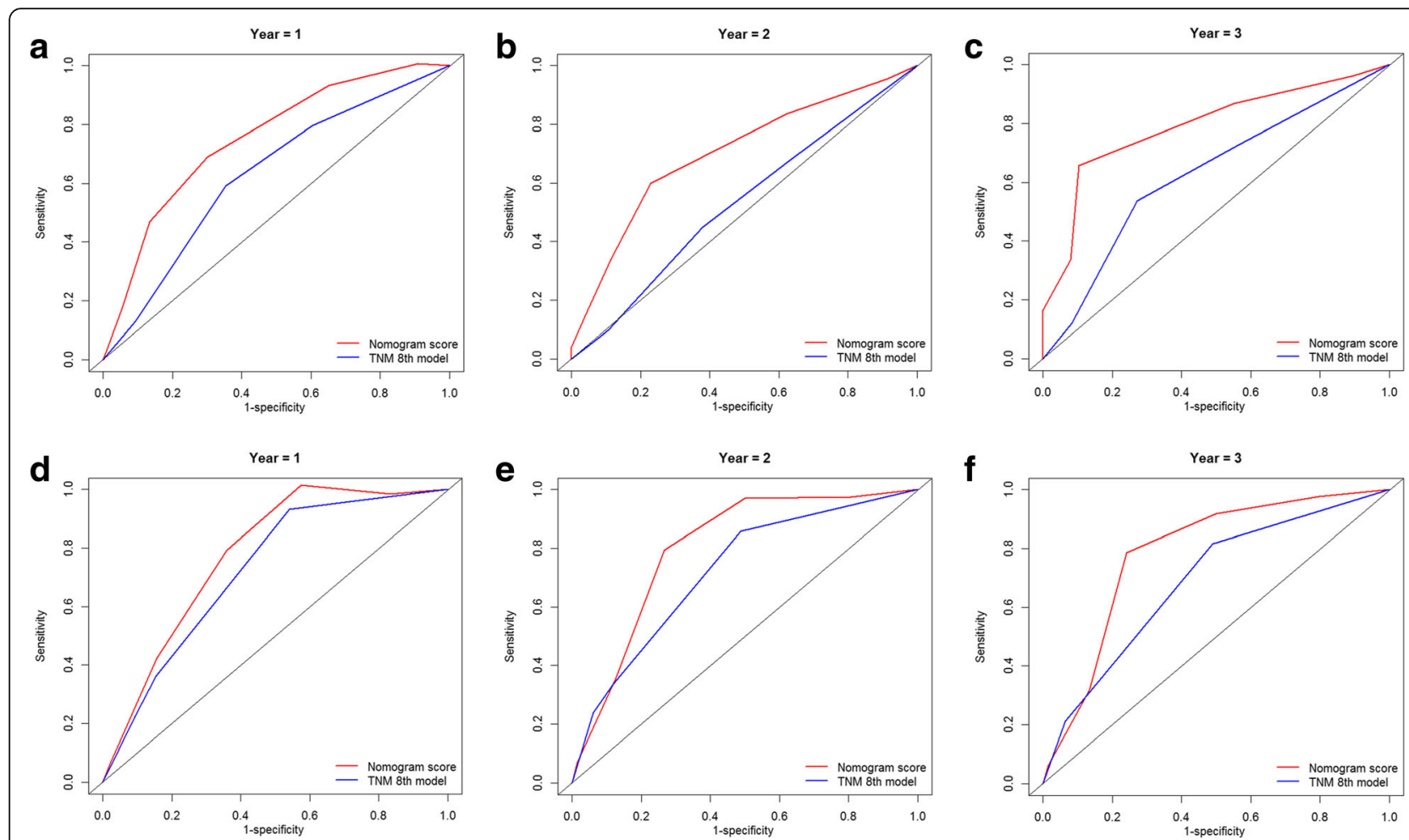

Fig. 6 Comparison of the ROC curves of the nomogram and the TNM stage system for 1-, 2- and 3-year CSS prediction in the train cohort $(\mathbf{a}, \mathbf{b}, \mathbf{c})$ and validation cohort (d, e, f). Abbreviations: CSS, cancer-specific survival; TNM, Tumor-Node-Metastasis

indicate inferior survival in our study, which could partly explain why the loss of monotonous gradient for survival prediction of TNM stage and the superior predictive power of the established nomograms in our study.

With the increasing occurrence and concern of competing risk events, more and more focuses have been paid on competing analyses, such as lung cancer [21], breast cancer [26] and gastric cancer [27]. Considering the non-cancer events contributed to $16.9 \%$ of deaths, competing interests were taken into account in survival analyses in this study. As far as we know, it was the first time to build prognostic nomograms to specially predict OS and CSS for patients with CHCC after surgery based on competing risk analysis. Significantly elevated predictive power was observed for the established nomograms in this study. The inclusion of additional variables guaranteed that nomograms were better in predicting OS and CSS, compared with the 8th edition TNM stage system. In addition, the nomograms were established based on a population-based dataset and cross-validated from an external dataset, making our results more generable than those from studies of small cohort or single center. Thus, a diverse range of parameters of $\mathrm{CHCC}$ patients are assessed by doctors more objectively and precisely based on the established nomograms. In addition, this newly established system can be used to identify subgroups of patients with a more homogeneous prognosis, estimate individual survival, and then to specialize personal treatment.

There were several limitations for this study. The major limitation of the present study is that not all risk factors were included to construct the nomograms. Some important tumor biomarker, such as carbohydrate antigen 19-9 (CA19-9), and some positive prognostic variables, such as surgical margin status and vascular invasion, were unavailable in SEER dataset. Maybe the additional inclusion of these variables might elevate the predictive power. This is also the major part of our future research. Another limitation is that although the established nomograms showed good discrimination and validation, the values of C-index and AUC are not relatively high. Further validation based on large-scale cohort is needed for these nomograms.

\section{Conclusion}

In conclusion, competing risk analyses were conducted and nomograms specially to predict OS and CSS for these patients were established for the first time in this study. The established nomograms can be used to accurately provide valuable prognostic information, allowing tailed treatments for patients with CHCC after surgery. 


\section{Abbreviations}

AUC: Area under ROC curve; CA19-9: Carbohydrate antigen 19-9; CHCC: Combined hepatocellular cholangiocarcinoma; Cl: Confidence interval; CIF: Cumulative incidence function; C-index: Concordance index; CSS: Cancer-specific survival; HCC: Hepatocellular carcinoma; HR: Hazard ratio; ICC: Cholangiocarcinoma; LN: Lymph node; OS: Overall survival; ROC: Receiver operating characteristic; SEER: Surveillance, Epidemiology, and End Results; SYSUCC: Sun Yat-Sen University Cancer Center; TNM: Tumornode-metastasis

\section{Acknowledgements}

The authors would like to thank SEER for open access to the database.

\section{Funding}

This work was supported by the Sun Yat-sen University Grant for Medical Humanities Practice and Teaching (No. 23000-18008023). The funding bodies had no role in the design of the study and collection, analysis, and interpretation of data and in the writing of the manuscript.

\section{Availability of data and materials}

The dataset from SEER database generated and/or analyzed during the current study are available in the SEER dataset repository (https://seer.cancer.gov/). Another dataset from SYSUCC dataset has been validated by uploading the key raw data onto the Research Data Deposit public platform (http:// www.researchdata.org.cn), with the Approval Number as RDDA2018000870.

\section{Authors' contributions}

$\mathrm{CBH}$ and $\mathrm{YZ}$ contributed to this work equally. $\mathrm{XJL}$ was responsible for conception, design and quality control of this study. CBH, YZ and ZYC performed the study selection, data extraction, statistical analyses, and was major contributors in writing the manuscript. CBH and ZYC participated in studies selection and statistical analyses. CBH, YZ and ZYC contributed in classification criteria discussion. $\mathrm{CBH}$ and $\mathrm{YZ}$ contributed to the writing of manuscript. XJL reviewed and edited the manuscript respectively. All authors read and approved the final manuscript.

\section{Ethics approval and consent to participate}

This study was approved by the Institutional Review Board of Sun Yat-sen University Cancer Center. All procedures performed in studies involving human participants were in accordance with the ethical standards of the institutional and/or national research committee and with the 1964 Helsinki Declaration and its later amendments or comparable ethical standards. Written informed consent was obtained from individual participants included in the study. All authors signed authorization forms and received permission from SEER to access and use the dataset.

\section{Consent for publication}

Not applicable.

\section{Competing interests}

The authors declare that they have no competing interests.

\section{Publisher's Note}

Springer Nature remains neutral with regard to jurisdictional claims in published maps and institutional affiliations.

\section{Author details}

'Department of Hepatobiliary and Pancreatic Surgery, State Key Laboratory of Oncology in South China, Collaborative Innovation Center for Cancer Medicine, Sun Yat-sen University Cancer Center, Guangzhou 510060, China. ${ }^{2}$ State Key Laboratory of Ophthalmology, Zhongshan Ophthalmic Center, Sun Yat-sen University, Guangzhou, Guangdong 510060, China.

Received: 21 November 2018 Accepted: 20 February 2019 Published online: 27 February 2019

\section{References}

1. Bosman FT. HH: WHO classifcation of tumours of the digestive system, 4th edn. Lyon: International Agency for Research on Cancer; 2010.

2. Kassahun WT, Hauss J. Management of combined hepatocellular and cholangiocarcinoma. Int J Clin Pract. 2008;62(8):1271-8.
3. Yin X, Zhang BH, Qiu SJ, Ren ZG, Zhou J, Chen XH, Zhou Y, Fan J. Combined hepatocellular carcinoma and cholangiocarcinoma: clinical features, treatment modalities, and prognosis. Ann Surg Oncol. 2012;19(9): 2869-76.

4. Yap $A Q$, Chen CL, Yong CC, Kuo FY, Wang SH, Lin CC, Liu YW, Lin TL, Li WF, Millan CA, et al. Clinicopathological factors impact the survival outcome following the resection of combined hepatocellular carcinoma and cholangiocarcinoma. Surg Oncol. 2013;22(1):55-60.

5. Allen RA, Lisa JR. Combined liver cell and bile duct carcinoma. Am J Pathol. 1949;25(4):647-55.

6. Xu L, Peng ZW, Chen MS, Shi M, Zhang YJ, Guo RP, Lin XJ, Lau WY. Prognostic nomogram for patients with unresectable hepatocellular carcinoma after transcatheter arterial chemoembolization. J Hepatol. 2015; 63(1):122-30.

7. He CB, Lin XJ. Inflammation scores predict the survival of patients with hepatocellular carcinoma who were treated with transarterial chemoembolization and recombinant human type-5 adenovirus H101. PLoS One. 2017;12(3):e0174769.

8. He CB, Lao XM, Lin XJ. Transarterial chemoembolization combined with recombinant human adenovirus type $5 \mathrm{H} 101$ prolongs overall survival of patients with intermediate to advanced hepatocellular carcinoma: a prognostic nomogram study. Chinese J Cancer. 2017;36(1):59.

9. He C, Mao Y, Lao X, Li S, Lin X. Neutrophil-to-lymphocyte ratio predicts overall survival of patients with combined hepatocellular cholangiocarcinoma. Oncol Lett. 2018;15(4):4262-8.

10. He C, Mao Y, Wang J, Song Y, Huang X, Lin X, Li S. The predictive value of staging systems and inflammation scores for patients with combined hepatocellular cholangiocarcinoma after surgical resection: a retrospective study. J Gastrointest Surg. 2018;22(7):1239-50.

11. Tian MX, He WJ, Liu WR, Yin JC, Jin L, Tang Z, Jiang XF, Wang H, Zhou PY, Tao CY, et al. A novel risk prediction model for patients with combined hepatocellular-cholangiocarcinoma. J Cancer. 2018;9(6):1025-32.

12. Kim SH, Park YN, Lim JH, Choi GH, Choi JS, Kim KS. Characteristics of combined hepatocelluar-cholangiocarcinoma and comparison with intrahepatic cholangiocarcinoma. Eur J Surg Oncol. 2014;40(8):976-81.

13. Ma MC, Chen YJ, Chiu TJ, Lan J, Liu CT, Chen YC, Tien HH, Chen YY. Positive expression of Midkine predicts early recurrence and poor prognosis of initially resectable combined hepatocellular cholangiocarcinoma. BMC Cancer. 2018;18(1):227.

14. Schiergens TS, Lindenthaler A, Thomas MN, Rentsch M, Mittermeier L, Brand K, Kuchenhoff H, Lee S, Guba M, Werner J, et al. Time-dependent impact of age and comorbidities on long-term overall survival after liver resection. Liver Int. 2016;36(9):1340-50.

15. Szpakowski JL, Tucker LY. Causes of death in patients with hepatitis B: a natural history cohort study in the United States. Hepatology (Baltimore, Md). 2013;58(1):21-30.

16. Shindoh J, Kawamura $Y$, Kobayashi $Y$, Kiya $Y$, Sugawara $T$, Akuta $N$ Kobayashi M, Suzuki Y, Ikeda K, Hashimoto M. Platelet-albumin score as a sensitive measure for surgical risk prediction and survival outcomes of patients with hepatocellular carcinoma. J Gastrointest Surg. 2018;23(1): 76-83.

17. Noordzij M, Leffondre K, van Stralen KJ, Zoccali C, Dekker FW, Jager KJ. When do we need competing risks methods for survival analysis in nephrology? Nephrol Dial Transplant. 2013;28(11):2670-7.

18. Harrell FE Jr, Lee KL, Mark DB. Multivariable prognostic models: issues in developing models, evaluating assumptions and adequacy, and measuring and reducing errors. Stat Med. 1996;15(4):361-87.

19. Norris CM, Ghali WA, Saunders LD, Brant R, Galbraith D, Faris P, Knudtson ML. Ordinal regression model and the linear regression model were superior to the logistic regression models. J Clin Epidemiol. 2006;59(5):448-56.

20. Li D, Zhong C, Tang X, Yu L, Ding K, Yuan Y. Competing nomograms help in the selection of elderly patients with colon cancer for adjuvant chemotherapy. J Cancer Res Clin Oncol. 2018;144(5):909-23.

21. Zhou H, Zhang Y, Qiu Z, Chen G, Hong S, Chen X, Zhang Z, Huang Y, Zhang L. Nomogram to predict cause-specific mortality in patients with surgically resected stage I non-small-cell lung cancer: a competing risk analysis. Clin Lung Cancer. 2018;19(2):e195-203.

22. Chantajitr S, Wilasrusmee $C$, Lertsitichai P, Phromsopha N. Combined hepatocellular and cholangiocarcinoma: clinical features and prognostic study in a Thai population. J Hepato-Biliary-Pancreat Surg. 2006;13(6): $537-42$. 
23. Bergquist JR, Groeschl RT, Ivanics $T$, Shubert CR, Habermann EB, Kendrick ML, Farnell MB, Nagorney DM, Truty MJ, Smoot RL. Mixed hepatocellular and cholangiocarcinoma: a rare tumor with a mix of parent phenotypic characteristics. HPB (Oxford). 2016;18(11):886-92.

24. Moeini A, Sia D, Zhang Z, Camprecios G, Stueck A, Dong H, Montal R, Torrens L, Martinez-Quetglas I, Fiel Ml, et al. Mixed hepatocellular cholangiocarcinoma tumors: Cholangiolocellular carcinoma is a distinct molecular entity. J Hepatol. 2017;66(5):952-61.

25. Chu KJ, Lu CD, Dong H, Fu XH, Zhang HW, Yao XP. Hepatitis B virus-related combined hepatocellular-cholangiocarcinoma: clinicopathological and prognostic analysis of 390 cases. Eur J Gastroenterol Hepatol. 2014;26(2):192-9.

26. Lian M, Perez M, Liu Y, Schootman M, Frisse A, Foldes E, Jeffe DB. Neighborhood socioeconomic deprivation, tumor subtypes, and causes of death after non-metastatic invasive breast cancer diagnosis: a multilevel competing-risk analysis. Breast Cancer Res Treat. 2014;147(3):661-70.

27. Shin DW, Suh B, Park Y, Lim H, Suh YS, Yun JM, Cho B, Yang HK. Risk of coronary heart disease and ischemic stroke incidence in gastric cancer survivors: a Nationwide study in Korea. Ann Surg Oncol. 2018;25(11):3248-56.

Ready to submit your research? Choose BMC and benefit from:

- fast, convenient online submission

- thorough peer review by experienced researchers in your field

- rapid publication on acceptance

- support for research data, including large and complex data types

- gold Open Access which fosters wider collaboration and increased citations

- maximum visibility for your research: over $100 \mathrm{M}$ website views per year

At $\mathrm{BMC}$, research is always in progress.

Learn more biomedcentral.com/submissions 